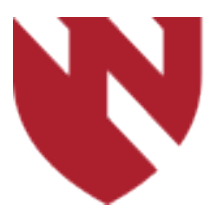

October 2021

\title{
Chronic Invasive Fungal Sinusitis due to Scedosporium Apiospermum causing Orbital Apex Syndrome
}

\author{
Dallin N. Christensen \\ University of Nebraska Medical Center \\ Christie Barnes \\ University of Nebraska Medical Center \\ Matthew Miller \\ University of Nebraska Medical Center
}

Tell us how you used this information in this short survey.

Follow this and additional works at: https://digitalcommons.unmc.edu/gmerj

Part of the Otolaryngology Commons

\section{Recommended Citation}

Christensen, D. N., Barnes, C., , Miller, M. Chronic Invasive Fungal Sinusitis due to Scedosporium Apiospermum causing Orbital Apex Syndrome. Graduate Medical Education Research Journal. 2021 Oct 04; 3(1).

https://digitalcommons.unmc.edu/gmerj/vol3/iss1/8

This Case Report is brought to you for free and open access by DigitalCommons@UNMC. It has been accepted for inclusion in Graduate Medical Education Research Journal by an authorized editor of DigitalCommons@UNMC. For more information, please contact digitalcommons@unmc.edu. 


\title{
Chronic Invasive Fungal Sinusitis due to Scedosporium Apiospermum causing Orbital Apex Syndrome
}

\author{
Abstract \\ Abstract \\ Introduction: \\ Chronic invasive fungal sinusitis (CIFS) is defined as fungal invasion of the sinonasal submucosa for \\ greater than 12 weeks. Common causes are dematiaceous molds followed by Aspergillus species, but \\ can rarely be caused by other pathogens \\ Case:
}

A 71-year-old immunocompetent male presented with orbital apex syndrome found to be due to chronic invasive fungal sinusitis caused by Scedosporium apiospermum. After surgical debridement and appropriate systemic antifungal therapy, he made a near full recovery.

Discussion:

Scedosporium apiospermum is an emerging pathogen and a rare cause of chronic invasive fungal sinusitis. The diagnostic and treatment dilemmas involved with this rare but emerging clinical entity will be discussed.

\section{Keywords}

Chronic invasive fungal sinusitis, invasive fungal sinusitis, rhinology, orbital apex syndrome, endoscopic sinus surgery, scedosporium apiospermum

\section{Creative Commons License} (c) (i) (9)

This work is licensed under a Creative Commons Attribution-Noncommercial-No Derivative Works 4.0 License. 


\section{Chronic Invasive Fungal Sinusitis due to Scedosporium apiospermum causing Orbital Apex Syndrome \\ Dallin N. Christensen ${ }^{1}$, Christie Barnes ${ }^{1}$, Matthew Miller ${ }^{1}$}

${ }^{1}$ University of Nebraska Medical Center, College of Medicine, Department of Otolaryngology - Head and Neck Surgery

https://doi.org/10.32873/unmc.dc.gmerj.3.1.007

\section{Abstract}

Chronic invasive fungal sinusitis (CIFS) is defined as fungal invasion of the sinonasal submucosa for greater than 12 weeks. Common causes are dematiaceous molds followed by Aspergillus species, but can rarely be caused by other pathogens. A 71-year-old immunocompetent male presented with orbital apex syndrome found to be due to chronic invasive fungal sinusitis caused by Scedosporium apiospermum. After surgical debridement and appropriate systemic antifungal therapy, he made a near full recovery. Scedosporium apiospermum is an emerging pathogen and a rare cause of chronic invasive fungal sinusitis. The diagnostic and treatment dilemmas involved with this rare but emerging clinical entity will be discussed.

\section{Introduction}

Fungal sinusitis is commonly categorized as either invasive or noninvasive disease. Invasive fungal sinusitis has been subcategorized as acute fulminant, granulomatous, and chronic invasive disease. ${ }^{1}$ Chronic invasive fungal sinusitis (CIFS) is defined as fungal invasion of the sinonasal submucosa with disease present for greater than 12 weeks. ${ }^{1}$ When compared to acute invasive disease, CIFS more insidious in its presentation, with symptoms developing over weeks to months, but can have devastating neurovascular consequences. CIFS is classically associated with diabetes mellitus and can lead to cranial neuropathies. ${ }^{1-3}$ It is commonly caused by dermatiaceous molds or aspergillus, and treatment typically consists of surgical debridement and antifungals such as amphotericin. Scedosporium is a rare pathogen found in soil, sewage, and polluted water. ${ }^{4}$ Though an uncommon cause of disease in immunocompetent individuals, it can be problematic due to resistance to amphotericin, fluconazole, and many other commonly utilized antifungals. ${ }^{3}$

Orbital apex syndrome describes the confluence of symptoms from deficits of the optic (II), oculomotor (III), trochlear (IV), ophthalmic (V1), and abducens nerves (VI). It is caused by a lesion at or near the orbital apex. It commonly presents with ophthalmoplegia and hypesthesia of the upper hemiface and may be distinguished from superior orbital fissure syndrome by the presence of decreased visual acuity.
We report a case of CIFS and orbital apex syndrome due to Scedosporium apiospermum in an immunocompetent male.

\section{Case}

A 71-year-old immunocompetent male presented with a 3-month history of unilateral headache and two weeks of diplopia. His headache involved his left facial, retro-orbital, parietal, and occipital regions, and was associated with light sensitivity and nausea. He had previously been seen by ENT at an outside facility and underwent debridement of a mucopyocele of the sphenoid sinus, balloon sinoplasty of the left sphenoid, bilateral maxillary antrostomies, and inferior turbinate reductions approximately two months prior to presentation. He initially experienced headache relief, but symptoms recurred within a week. Several weeks later, he developed

A

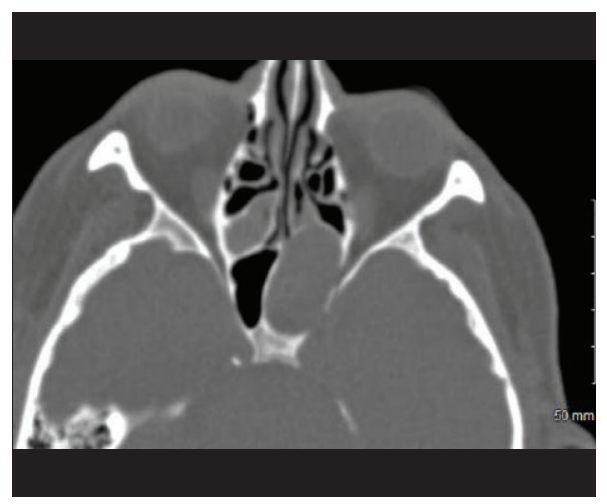

C

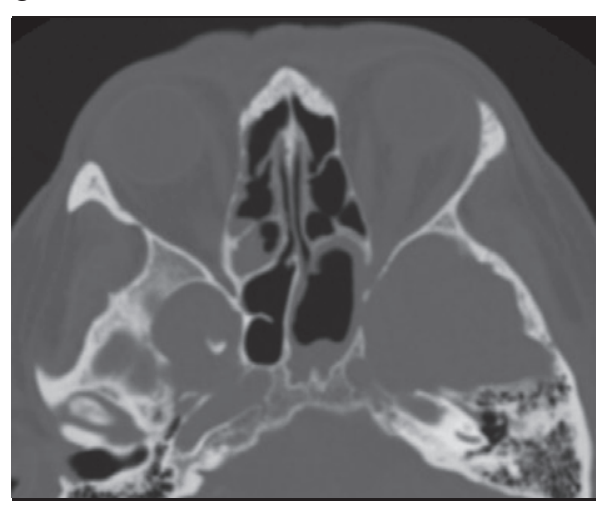

diplopia which was worse with left gaze and subsequently sought further care.

On physical exam, he was noted to have left eye ptosis, lateral and upward gaze limitations, afferent pupillary defect, and decreased visual acuity, as well as decreased sensation in the left V1 distribution.

CT scan was significant for soft tissue fullness of the left anterior cavernous sinus, supraorbital fissure, and orbital apex, as well as moderate scattered mucosal thickening with variegated material (Fig. 1). Follow up MRI imaging confirmed fullness and enhancement of the cavernous sinus, supraorbital fissure, and orbital apex, without evidence of leptomeningeal involvement (Fig. 2).

The patient was started on amphotericin B and broad-spectrum antibiotics and taken to the OR where he underwent left posterior

B

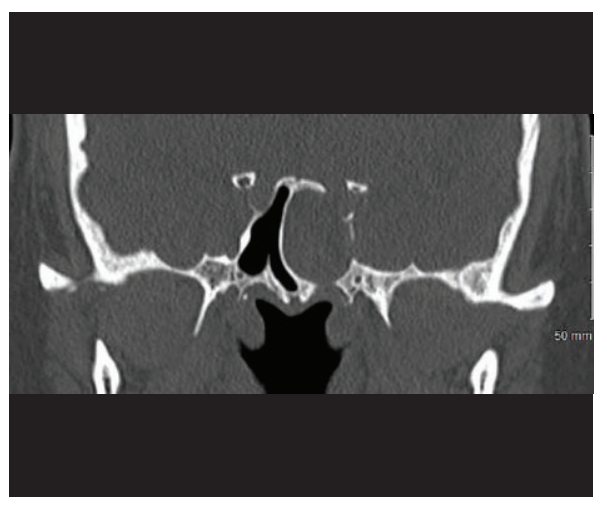

D

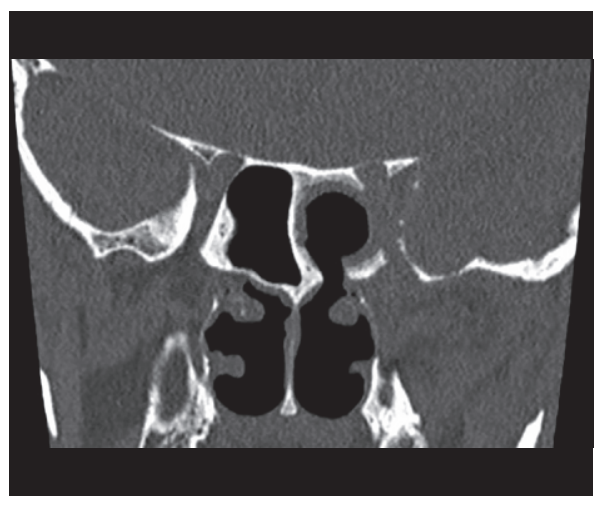

Figure 1. Evolving bony destruction at the orbital apex and cavernous sinus. A and B: Computed tomography scan of the patient's sinuses without contrast 2 months prior to presentation to our medical center. Axial and coronal views are shown, respectively. White arrows show areas of bony destruction along the orbital apex while black arrows mark bony dehiscence along the cavernous sinus. C and D: Computed tomography scan of the patient's sinuses without contrast at presentation to our medical center. Worsening bony destruction is seen. 
ethmoidectomy, left sphenoidotomy, and bilateral maxillary washout. Intraoperative findings were significant for thickened, yellow sphenoid mucosa with extension toward the pterygopalatine fossa and orbital apex, with complete erosion of the bone overlying the left carotid, cavernous sinus, and orbital apex. Biopsies of the abnormal mucosa were taken, and histological analysis demonstrated branching septate hyphae invading the submucosa with focal areas of necrotizing inflammation. Fungal cultures were positive for Scedosporium apiospermum and all other cultures remained negative.

The final diagnosis was chronic invasive fungal sinusitis with orbital apex syndrome due to Scedosporium apiospermum infection. The patient did not undergo further surgical debridement due to the location of invasion. He was transitioned from amphotericin B to voriconazole and terbinafine. He had an uncomplicated post-operative course in which he experienced resolution of his headaches and was discharged on oral voriconazole. At six week follow up, the patient had continued relief of headache, and had interval improvement in his cranial nerve deficits.

Verbal consent to use details from this case for educational and academic purposes was given by the patient.

\section{Discussion}

To our knowledge, this is the third case of chronic invasive fungal sinusitis secondary to Scedosporium apiospermum infection in the literature. Khoueir et al report a similar case in a 72-year-old man who had sphenoidal disease, bilateral abducens palsy, and an extradural abscess. ${ }^{4} \mathrm{He}$ refused antifungal medical therapy and was successfully treated with surgical debridement alone. Montone et al also idented a case of CIFS due to Scedosporium apiospermum in a review of 400 patients with fungal rhinosinusitis but did not provide specific details about the case. ${ }^{5}$ Of note, both these cases and the case presented here occurred in immunocompetent individuals.

In addition to surgical debridement, treatment of CIFS typically includes correction of any underlying immunodeficiency and empiric antifungal treatment, frequently with amphotericin. This is potentially problematic in the case of invasive Scedosporium apiospermum disease as amphotericin frequently has limited activity against Scedosporium species. ${ }^{3}$ Echinocandins such as caspofungin and micafungin, as well as fluconazole also lack efficacy against Scedosporium species. Voriconazole and
A

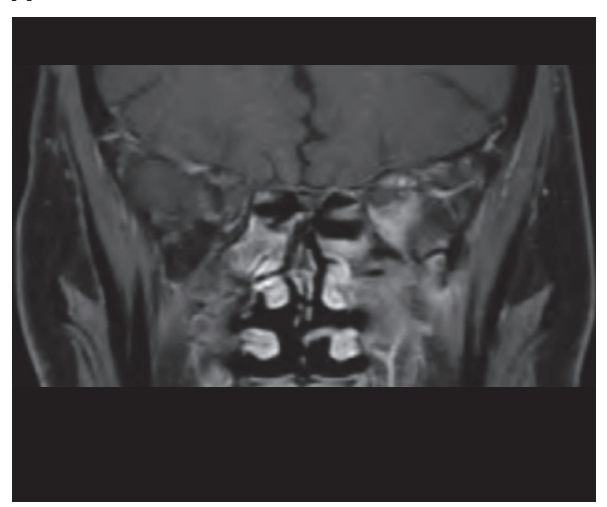

C

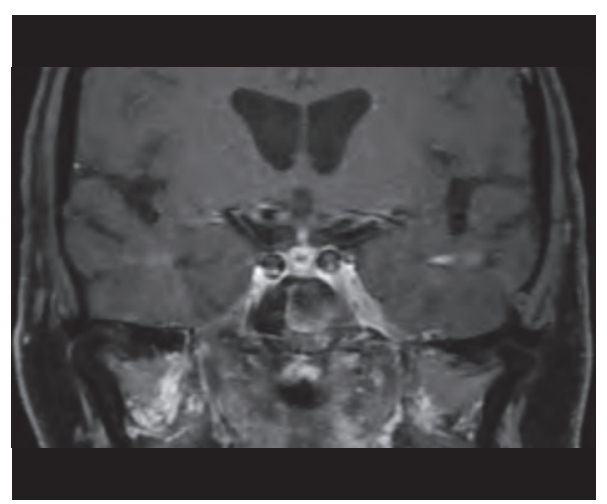

B

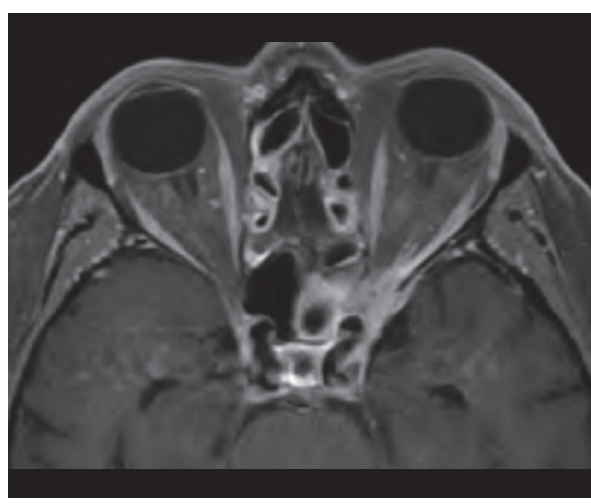

D

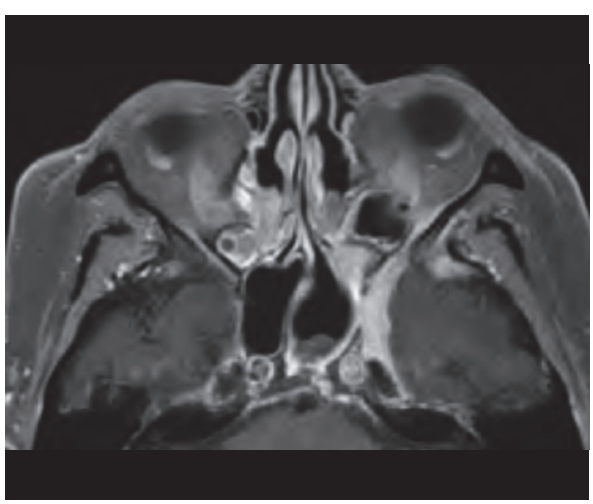

Figure 2. Magnetic Resonance Imaging showing enhancement at the orbital apex and cavernous sinus. All sequences are T1 weighted, post-contrast images. A and B: Coronal and axial images, respectively, of the orbital apex, non-specific enhancement is seen. $C$ and $\mathrm{D}$ : Coronal and axial images, respectively, of the cavernous sinus. Non-specific enhancement is seen, without apparent leptomeningeal involvement.

posaconazole have good coverage against $S$. apiospermum. ${ }^{3}$ This highlights the utility of culture and culture-directed therapy.

\section{Conclusion}

The mainstays of treatment in CIFS are surgical debridement and antifungal treatment, typically with amphotericin. This case highlights the importance of culture directed therapy, as $S$. apiospermum is resistant to amphotericin. Optimum treatment of CIFS due to $S$. apiospermum remains unclear largely due to the rarity of the disease. However, in the two cases discussed, adequate control was seemingly obtained following surgical debridement alone in one, and surgical debridement plus voriconazole in the other.

\section{References}

1 DeShazo RD, O'Brien M, Chapin K, et al. "A new classification and diagnostic criteria for invasive fungal sinusitis." Archives of Otolaryngology-Head \& Neck Surgery, 123(11), (1997) : 1181-1188.

2 DeShazo RD. "Syndromes of invasive fungal sinusitis." Medical mycology 47.Supplement_1 (2009) S309-S314.

3 Thompson III, GR., and Patterson TF. "Fungal disease of the nose and paranasal sinuses." Journal of Allergy and Clinical Immunology 129.2 (2012): 321-326.

4 Khoueir, N, Verillaud B, and Herman P. "Scedosporium apiospermum invasive sinusitis presenting as extradural abscess." European annals of otorhinolaryngology, head and neck diseases 136.2 (2019): 119-121.

5 Montone, KT, Livolsi KT, Feldman VA et al. "Fungal rhinosinusitis: a retrospective microbiologic and pathologic review of 400 patients at a single university medical center." International Journal of Otolaryngology 2012 (2012). 